\title{
The value of theory of color in modern menswear design
}

\author{
Song Ting ${ }^{1, a}$ \\ ${ }^{1}$ Jiangxi Institute of Fashion Technology, Jiangxi, Nanchang, 330201 \\ a914284677@qq.com
}

Keywords: Fashion colour; Men's clothing design; The color; The value of

\begin{abstract}
Early after a study found that men's design for the use of color is less, it is hard to find in the men's fashion colour design material, most is the basis of the black and white and gray color. In recent years, the development trend of men's clothing design is diverse, the designer's creative thinking and expression methods due to the time change and innovation. In today's increasingly more popular colour elements in the men's clothing design elements are important, color in men's clothing design favored by domestic and foreign clothing designers. From the point of some men's clothing enterprise, men's success or failure of product design also can be determined by men's wear color design.
\end{abstract}

\section{Introduction}

Clothing is made up of profile type, color and material of three elements, they have a practical significance, namely more aesthetic significance. The colour element the most eye-catching, intuitive, vivid, and the most remarkable. Clothing color is an important content in the clothing design, costume designer in the design must consider the factors in the clothing a object, the environment, with fashion elements, etc. And the color in the clothing products are not only play the role of a decoration, colour can affect the person's spirit will more, cheerful mood. Has certain social symbolic clothing color, also is a kind of silent language, the most intuitive reflect the person's feelings, an era of civilization and social culture. Each color has its specific connotation and show the character and emotion are very rich and full of all kinds of changes.

In today's clothing product design, development and sales of the real time people's aesthetic demand and clothing products is very necessary, the application of color became extremely important, trendy fashion and popular clothing color combination application of the increasingly close. So for the color characteristics and cycle analysis carefully, and real-time to use in the design of the men's clothing is a fashion designers have, only in this way can keep up with the pace of the international fashion and become the leader of the market.

\section{The concept of color}

Color refers to the color in a wide range, within the prescribed range or a time interval, the design of products in consumers' demand in different lightness so popular. Several or several groups of different purity color and tonal, can be in a developed city or region of color for short. If these colors, color through the institute of professional color or international committee for approval, can be released around the world, this issue can be referred to as international fashion colour. The shortest, the most sensitive period of time is a characteristics of color. A sign of human civilization is the fashion colour, it is permeated with the youth melody, is the most beautiful and good human spirit to enjoy, like social popular things in life, color is a kind of psychological feeling of human society a thing of beauty. Fashion colour, English: FashionColour, "fashion", is favored by the public, like popular application of a set of color, have a certain degree of fashion, and it is within a certain time season and region. Also is in a period of time, a certain social politics, economy, environment, culture and human psychological activities under the factors such as comprehensive.

\section{The value of the color}

In the domestic clothing market increased in recent years for the research and application of color, because the color is more and more consumer attention by enterprises, and has to do multiple areas. 
Many fields, such as the development of the color will be the future an important growth point of economy, has become the industry consensus. Scientific application and understanding of color, in the development of textile and clothing industry perform to the best of the situation, has high performance proves that the color of commercial value. In the development of some clothing very good city to conduct market research found that on the fashion colour application the word "color" is a very popular vocabulary. People buy clothes in this behaviour in the attention to color, has become a kind of fashion of modern life.

Fashion colour can optimize the design of the clothing products, improve the prices of clothing products and sales in the market, strengthen the competitiveness of the products, according to the sales promotion the brand image and reputation. Fashion colour promote people's cultivation of costume culture and color, color culture, rich vision, increase employment opportunities, driving the development of related industries to extend fashion colour development.

\section{Fashion colour in the application of modern menswear design}

In the modern men's clothing design, popular element and color already appear particularly important. From the domestic and foreign markets, the same specification size, style of men's clothing styles, fabrics in gas non-mainstream color period, at the prices and the mainstream color peak can be quite a few times, not a mainstream style and colour men become "bargains" and cut processing, men's goods also gradually reflect its strong "time value" potential, fashion colour application and research in the men's clothing design is the soul of the era design considerations.

The color in the design of modern men's clothing. The combination of color and basic color. In men's wear colour collocation design in addition to the hue, lightness, purity, and color harmony rule, the color selection is also very important. Basic color refers to the inherent in the traditional sense of men's colour fundamental key, such as black, grey, white, no color and low Ming dull color such as blue, brown, low Gao Mingqing color, good pure light color, low bright pure color and other commonly used color; In today's men's wear color design makes the combination of the most widely used method, such as with the use of tendency of the color in color chromatography, bottoms USES basic common color. But also men's coat is a foundation, and clothing with popular color, the design can be more cause the resonance of the consumers, the effect of design is both can reflect a certain sense of fashion, and can be compared with the more conservative man accepted market consumers, make the sells men's clothing has more consumers.

Single color selection and application. In the design of color now single color selection and application are also very popular. In men's clothing design monochrome dress more and more concern very much, in the newly released a set of color, each color can be a single application in the design, and every single color choice in men's clothing design can produce new colors. In men's wear colour collocation, the same kind of clothing styles selected single falling tone color processing, by lowering the brightness, purity, and get the similar color of the same color, also widely used in the men's clothing design.

The separation of color combination. Under the premise of keeps the same color trend, by changing the popular colour change of lightness and purity to derive more specific series color, such as using the base color, single color, black and white ash as the main tonal, only used on local and accessories fashion colour as the ornament can foil a feeling of men's masculine and bold.

The integral design of the color with men's clothing. Are the main differences between men's and women's clothing in the use of color to highlight the era of male image in design. A careful study on the basis of published color to garment colour, find the colour on the fashion color and don't compare a matching color fabrics, combined with the design draft pick out several colors to match colors plan, for a variety of color combinations on the same garment color matching practice, commonly used "set the color to finalize the design" and the design of the tone "setting". Issued in accordance with the trend of fashion colour, the color of several groups or dozens of kinds of colour, design is when the design should consider the following questions:

First, should choose when needed in the design of color matching just released at the beginning of the hair color and the color, is common in the market of color. If in the men's clothing design are 
applied to the design and color of colored fabrics should be in the fabric of choice is to choose the base of color is the color or color fabrics Or there is a color digital printing design, as shown in figure 1.

Second, use color contrast color or complementary color processing, decorated a few on clothes as far as possible, using pure color with tall lightness, strengthen contrast, small area using these colors is called color ornament, this method can reflect the fashion colour of colour aesthetic feeling, as shown in figure 2 .

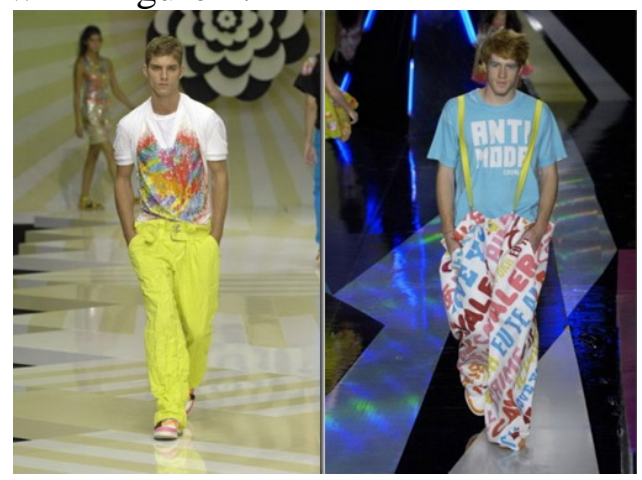

Fig.1 Design of men's clothing design

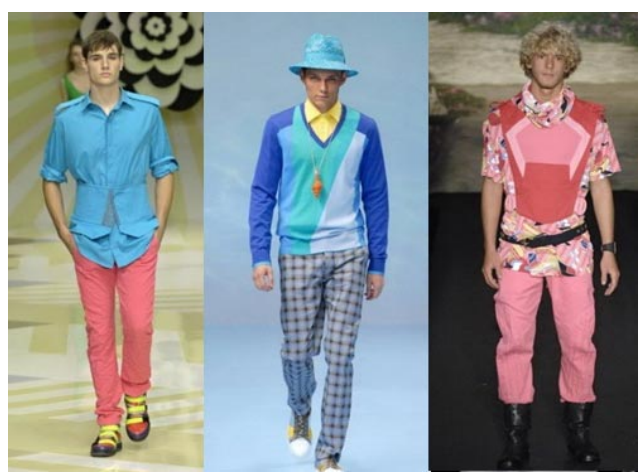

Fig. 2 The ornament color menswear design

Third, in order to make the color effect garments' integral design is reasonable and comfortable good-looking and have administrative levels feeling, should choose the right threads commonly used color or ash is commonly used color as harmonic color, can produce the effect of lasting able to bear or endure look.

Fourth, the fashion colour large scale application. In the fashion of men's clothing design, such as t-shirts, checkered shirt $60 \%$ color should be used as the dominant color, $40 \%$ used commonly used color, basic color, make whole clothing with modern neutral aesthetic feeling.

Fifth, in the clothing fashion colour in small proportion. On some fixed formal clothing, such as baseball jacket, recreational and west clothing can use most of $70 \%$ above is commonly used color, the other $30 \%$ is the use of color, people can be seen everywhere in life widely used is commonly used in the clothing area using of color, the color is the most commonly used color fashion ornament, enhance contrast using small area, this application can produce unique and unexpected effect, can make the garment more specific, highlight the administrative levels and to strengthen the fashion colour compared with commonly used color to let a person shine at the moment.

Sixth, in the men's wear color design, control of the color is very important, such as can be falling tone color group released the color processing will receive the effect of the wasted effort, such as purity of falling tone, falling tone lightness processing, at the same time should be considered with the area of the clothing color using a tonal color trend in men's clothing design style is what color.

Above all is how to apply in the design of men's fashion colour, flexible, should not blindly follow, should be combined with the specific design, sales area factors such as comprehensive consideration, is men's clothing color should pay attention to in practice.

\section{Conclusion}

Fashion colour in the contemporary society is people attention and accepted, the use of color in men's clothing design can't hidebound, should use the present popular color design clothing. The future world is rich and colorful, not only is the clothing industry, and other industries demand can not lack of fashion colour, for fashion colour in the application of men's clothing design should now continue to delve into, to beautify the life of people, improve people's quality of life.

\section{Reference}

[1] Dapeng Yuan. Theory of color in the clothing design of prediction and expression [J]. Journal of information science and technology of China, 2005, 8. 
[2]Shujuan Li. Theory of color design of children's clothing products [J]. Journal of jiyuan vocational and technical college, 2010, 3.

[3] Jianwu Xia. Introduction to the application of fashion colour in children's clothing design [J]. Journal of wenzhou university, 2004, 4.

[4] Yuzheng Lu, Spring and summer dress fashion colour hierarchical clustering method [J]. Journal of textile, 2015, 10. 